\title{
Simulation of air-water interfacial mass transfer driven by high-intensity isotropic turbulence
}

\author{
H. HERLIN A ${ }^{1} \dagger$, J. G. W I S S I N K ${ }^{2}$, \\ ${ }^{1}$ Institute for Hydromechanics, Karlsruhe Institute of Technology, Kaiserstr.12, \\ 76131 Karlsruhe, Germany \\ ${ }^{2}$ Department of Mechanical and Aerospace Engineering, Brunel University London, Kingston \\ Lane, Uxbridge, UB8 3PH, UK
}

(Received ?; revised ?; accepted ?. - To be entered by editorial office)

Previous direct numerical simulations (DNS) of mass transfer across the air-water interface have been limited to low-intensity turbulent flow with turbulent Reynolds numbers of $R_{T} \leqslant 500$. This paper presents the first DNS of low-diffusivity interfacial mass transfer across a clean surface driven by high-intensity $\left(1440 \leqslant R_{T} \leqslant 1856\right)$ isotropic turbulent flow diffusing from below. The detailed results, presented here for Schmidt numbers $S c=20$ and 500, support the validity of theoretical scaling laws and existing experimental data obtained at high $R_{T}$. In the DNS, to properly resolve the turbulent flow and the scalar transport at $S c=20$ up to $524 \times 10^{6}$ grid points were needed, while $65.5 \times 10^{9}$ grid points were required to resolve the scalar transport at $S c=500$, which is typical for oxygen in water. Compared to the low $R_{T}$ simulations, where turbulent mass flux is dominated by large eddies, in the present high $R_{T}$ simulation the contribution of small eddies to the turbulent mass flux was confirmed to increase significantly. Consequently, the normalised mass transfer velocity was found to agree with the $R_{T}^{-1 / 4}$ scaling, as opposed to the $R_{T}^{-1 / 2}$ scaling that is typical for low $R_{T}$ simulations. At constant $R_{T}$, the present results show that the mass transfer velocity $K_{L}$ scales with $S c^{-1 / 2}$, which is identical to the scaling found in the large-eddy regime for $R_{T} \leqslant 500$. As previously found for a no-slip interface, also for a shear-free interface the critical $R_{T}$ separating the large from the small eddy regime, was confirmed to be about $R_{T}=500$.

Key words:

\section{Introduction}

This paper considers the transport of low soluble atmospheric gases, such as oxygen, across the air-water interface promoted by high-intensity isotropic turbulence diffusing from below. Because of the very low mass diffusivity of such gases in water, in combination with the high-intensity turbulence, the corresponding concentration boundary layer thickness at the surface is significantly smaller than the Kolmogorov length scale of the turbulent flow (e.g. Jähne \& Haussecker 1998; Brumley \& Jirka 1988). Hence, an exceedingly fine resolution is needed to fully resolve the scalar transport dynamics. The latter is extremely challenging both when performing numerical simulations (see e.g. Rodi 2017) as well as laboratory experiments. Consequently, previously performed direct numerical

$\dagger$ Email address for correspondence: herlina.herlina@kit.edu 
simulations (DNS) and large-eddy simulations (LES) were limited to low turbulence intensities. The DNS were usually confined to relatively low Schmidt numbers, $S c=\nu / D$, where $\nu$ is the kinematic viscosity and $D$ is the mass diffusivity, (e.g. Schwertfirm \& Manhart 2007; Handler et al. 1999; Khakpour et al. 2011; Yang \& Shen 2017). High Schmidt number DNS were performed for buoyancy-induced mass transfer by Fredriksson et al. (2016) and Wissink \& Herlina (2016) with surface heat fluxes of $100 \mathrm{~W} / \mathrm{m}^{2}$ and up to $\sim 1600 \mathrm{~W} / \mathrm{m}^{2}$, respectively, and for isotropic-turbulence induced mass transfer by Herlina \& Wissink $(2014,2016)$. High Schmidt number LES was e.g. performed by Magnaudet \& Calmet (2006) for open-channel flow, while Hasegawa \& Kasagi (2008) carried out a hybrid DNS-LES of surface-shear driven mass transfer. Similarly, present experimental techniques still face difficulty in properly resolving the instantaneous scalar and flow properties within the concentration boundary layer (Chu \& Jirka 1992; Atmane \& George 2002; Variano \& Cowen 2013). Hence, only in a limited number of experiments (e.g. Herlina \& Jirka 2008; Janzen et al. 2010) sufficiently detailed simultaneous measurements within the concentration boundary layer for varying grid-stirred turbulent intensities were reported.

The present DNS was motivated by the need for highly-accurate unbiased data in the high $R_{T}$ regime to further investigate the dynamics of mass transfer across a shear-free surface dominated by small-scale energy-dissipating turbulent motions (cf. Theofanous et al. 1976; Theofanous 1984) that need to be fully resolved by the numerical mesh. These new results complement our previous DNS results obtained for low to moderate $R_{T}$, where mass transfer was driven by larger scale energy-containing turbulent motions. Hence, both the large and small-eddy dominated regimes for isotropic-turbulence driven mass transfer across a shear-free surface are covered. Note that for severely-contaminated interfaces - modeled using a no-slip surface boundary condition - both regimes were already explored in Herlina \& Wissink (2016), hereafter referred to as HW16.

The rate of mass transfer across the air-water interface is measured by the global transfer velocity

$$
K_{L}=\frac{|j|}{c_{s}-c_{b}},
$$

where $j$ is the interfacial mass flux and $c_{s}$ and $c_{b}$ are the average gas concentrations at the surface and in the bulk (well-mixed region), respectively. All conceptual models assume that the mass transfer is determined by no more than one length scale and one time scale (Brumley \& Jirka 1988). Many empirical relations between $K_{L}$ and measurable flow quantities have been suggested in the past, ranging from the film model of Lewis \& Whitman (1924) to Danckwerts' surface renewal model (Danckwerts 1951). Lewis and Whitman assumed the presence of stagnant films on both sides of the interface where molecular diffusion controls mass transfer, giving $K_{L}=D / \delta_{*}$, where $\delta_{*}$ is the film thickness. The surface renewal model reads

$$
K_{L} \approx \sqrt{D r},
$$

where $r$ is the surface renewal rate. The main problem for this model is the specification of the applicable renewal rate, which either requires direct determination or modelling. Fortescue \& Pearson (1967) suggested that $r$ is determined by the time-scale associated with the largest turbulent eddies, while Banerjee et al. (1968) and Lamont \& Scott (1970) used the time-scale of the small eddies.

In the large-eddy model, $r$ is usually determined using characteristic turbulent velocity $\left(u_{\infty}\right)$ and length $(\Lambda)$ scales, resulting in

$$
K_{L} \approx \sqrt{D u_{\infty} / \Lambda} .
$$


On the other hand, in the small-eddy model $r=\sqrt{\epsilon / \nu}$ is calculated using the Kolmogorov scales, where $\epsilon$ is the turbulent dissipation rate, giving

$$
K_{L} \approx \sqrt{D}(\epsilon / \nu)^{1 / 4}
$$

Theofanous et al. (1976) performed a dimensional analysis and subsequently proposed to combine the large and small-eddy models after concluding that the large eddies are most important for the smaller turbulent Reynolds numbers $R_{T}$ - defined in (4.2) below - while the small eddies become important for large $R_{T}$. The critical turbulent Reynolds number dividing the two regimes was found to be $R_{T} \approx 500$.

As mentioned above, because of limited computational resources, previous numerical work on interfacial gas transfer has been limited to relatively low turbulent Reynolds numbers and/or low Schmidt numbers. In this paper, we present the results of a direct numerical simulation performed for a range of high turbulent Reynolds numbers $\left(1440 \leqslant R_{T} \leqslant 1856\right)$ and Schmidt numbers of $S c=20$ and 500, where the latter is characteristic for the transport of atmospheric gases dissolved in water. The present $R_{T}$ is significantly larger than the critical $R_{T}$ such that the Kolmogorov time-scale will determine the interfacial mass transfer dynamics. The new high $R_{T}$ results will be compared to our previous low to moderate $R_{T}$ results from Herlina \& Wissink (2014), hereafter referred to by HW14.

\section{Numerical Method}

The fluid motion is determined by solving the non-dimensional incompressible NavierStokes equations, where the continuity equation reads

$$
\frac{\partial u_{j}}{\partial x_{j}}=0
$$

and the momentum equations are given by

$$
\frac{\partial u_{i}}{\partial t}+\frac{\partial u_{i} u_{j}}{\partial x_{j}}=-\frac{\partial p}{\partial x_{i}}+\frac{1}{R e}\left(\frac{\partial^{2} u_{i}}{\partial x_{j} \partial x_{j}}\right) \quad \text { for } \mathrm{i}=1,2,3,
$$

where $j=1,2,3, x_{1}=x, x_{2}=y$ are the horizontal directions and $x_{3}=z$ is the vertical direction, $u_{1}=u, u_{2}=v$ and $u_{3}=w$ are the velocities in the $x, y$, and $z$ directions, $p$ is the pressure and $t$ denotes time. $R e=U L / \nu$ is the Reynolds number, where $U, L$ are reference velocity and length scales, respectively. Note that in the absence of mean shear, to characterise the turbulent flow, it would be more appropriate to use a turbulent Reynolds number $R_{T}$, see (4.2). However, the characteristic turbulent velocity and length scales used to calculate $R_{T}$ can only be determined after the computation and are basically time dependent owing to the short simulation time. Hence, $U$ and $L$ are used as place holders for the non-dimensionalisation. In our case, where $R e=1200$ (cf. $\S 3$ ), a convenient choice would be e.g. $U=12 \mathrm{~cm} / \mathrm{s}, L=1 \mathrm{~cm}$ and $\nu=10^{-6} \mathrm{~m}^{2} / \mathrm{s}$, which is relevant for the grid-stirred experiments of e.g. Herlina \& Jirka (2008). In the subsequent analysis, most of the results are renormalised using the characteristic turbulent velocity and length scales.

The non-dimensional convection-diffusion equation that governs the transport of the dimensionless passive scalar $c^{*}=c^{*}(x, y, z, t)$ reads

$$
\frac{\partial c^{*}}{\partial t}+\frac{\partial u_{j} c^{*}}{\partial x_{j}}=\frac{1}{R e S c}\left(\frac{\partial^{2} c^{*}}{\partial x_{j} \partial x_{j}}\right),
$$


where

$$
c^{*}=\frac{c-c_{b, 0}}{c_{s}-c_{b, 0}},
$$

in which $c_{b, 0}$ is the initial concentration in the bulk and $c_{s}$ is the concentration at the surface, which is assumed to be fully saturated at all times. Hereafter the dimensionless concentration $c^{*}$ will be denoted by $c$. Note that the above implies that initially $c=1$ at the surface and $c=0$ in the bulk.

The present numerical simulation was performed using our solver that was specifically developed to accurately resolve low-diffusivity interfacial mass transfer. In the past, the code was extensively verified (e.g. Kubrak et al. 2013; Herlina \& Wissink 2014) and used to solve interfacial mass transfer problems driven by low to moderate levels of isotropic turbulence (Herlina \& Wissink 2014, 2016; Wissink et al. 2017) and by buoyantconvection (Wissink \& Herlina 2016). The 3D incompressible Navier-Stokes equations were solved using a fourth-order-accurate kinetic energy conserving discretisation of the convective terms (Wissink 2004) combined with a fourth-order central discretisation of the diffusive terms. The pressure Poisson equation was solved using the conjugate gradient method with a simple diagonal preconditioning. The second-order accurate AdamsBashforth method was used for time integration.

Together with the flow, convection-diffusion equations for two scalars were solved simultaneously. The fifth-order-accurate WENO scheme of Liu et al. (Liu et al. 1994) and the fourth-order-accurate central discretisation were used to discretise scalar convection and diffusion, respectively. A third-order Runge-Kutta method was used for the time integration. Because the scalar diffusivity can be more than two orders of magnitude smaller than the momentum diffusivity, a dual-meshing strategy was employed using a refined mesh for the scalar transport equations. The code was parallelised by dividing the mesh into a number of blocks of equal size. Communication between blocks was performed using the standard message passing interface (MPI) protocol. A more detailed description of the numerical method can be found in Kubrak et al. (2013).

\section{Computational setup}

A schematic of the computational domain is shown in figure 1. The interfacial mass transfer was modeled by a direct numerical simulation. The size of the DNS domain (upper box in figure 1) was $20 L \times 20 L \times 5 L$ in the $x, y, z$ directions, respectively ( $L$ is the reference length scale, cf. $\S 2$ ). To properly resolve the flow field and the scalar with $S c=20$ a $1024 \times 1024 \times 500$ base-mesh was used. For the scalar with $S c=500$, a refined mesh with $5120 \times 5120 \times 2500$ was employed. In the $x$ and $y$-directions a uniform mesh was used, while in the $z$-direction the mesh was stretched according to

$$
z(k)=z(0)+\left[\frac{\tanh \left(z_{\phi}\right)}{\tanh \left(z_{1}\right)}\right]\left(z\left(n_{z}\right)-z(0)\right)
$$

for $k=1, \ldots, n_{z}-1$, with $z_{1}=\theta / 2$ and $z_{\phi}=k z_{1} / n_{z}$ where $n_{z}$ is the number of nodes in the $z$-direction. The stretching is controlled by the parameter $\theta$, which is set to $\theta=4.7$. The grid resolution near the interface is such that (i) the local vertical grid size $\Delta z$ is about $12 \% L_{B}$ and (ii) the geometric mean of the grid-cells is less than $\pi L_{B}$, where $L_{B}$ is the Batchelor scale. The two conditions indicate that the Grötzbach criterion is fulfilled and the scalar distribution is well resolved (Grötzbach 1983).

As in our previous simulations, the isotropic turbulence introduced at the bottom of the computational domain was generated in a separate large-eddy simulation (LES) that ran concurrently with the DNS. To achieve a high turbulent Reynolds number $R_{T}$, 


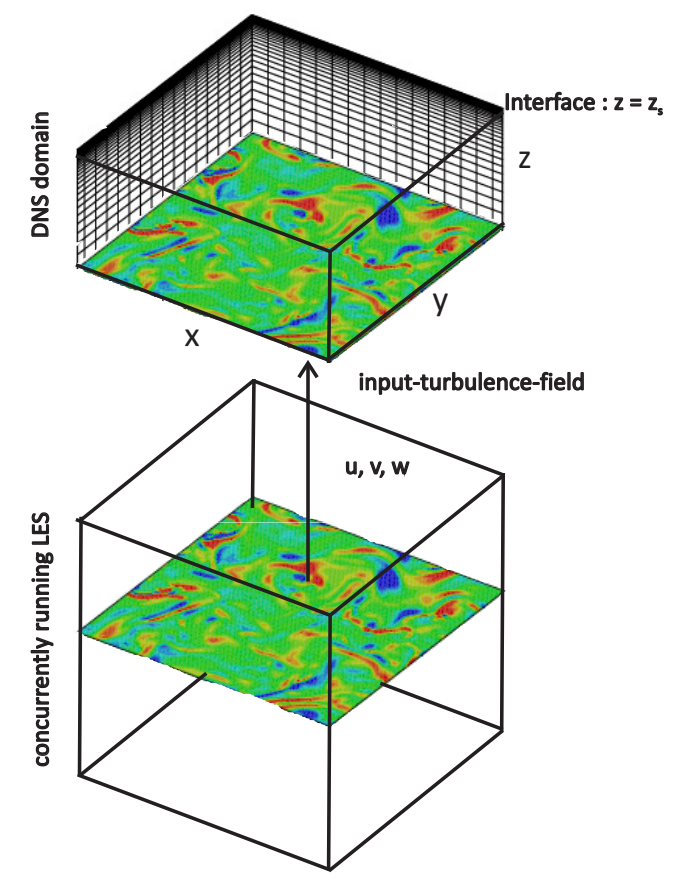

FIGURE 1. Schematic illustration of the computational domain.

a large $20 L \times 20 L \times 20 L$ LES domain was combined with a relatively high Reynolds number of $R e=1200$ and a turbulent kinetic energy level of $k=0.09375 U^{2}$ (see also $\S 2$ ). The LES box was discretised using a $256 \times 256 \times 256$ uniform mesh distributed over 512 processing cores. Periodic boundary conditions were employed for the velocities in all three directions. The subgrid scale turbulence was modeled using the standard Smagorinsky method with constant $C_{S m a g}=0.22$. Every time step the three components of the velocity field were rescaled so that the isotropy and the turbulence intensity were maintained.

In the DNS, the surface was assumed to remain flat at all times. For the velocity field, free-slip boundary conditions were employed at the surface, while periodic boundary conditions were used in the horizontal directions. At the surface, the scalar concentration was assumed to be at saturation $(c=1)$ at all times, while the concentration was initialised by

$$
c=\operatorname{erfc}\left(\zeta \sqrt{\frac{S c R e}{4 t^{\prime}}}\right)
$$

where $\zeta$ is the distance to the surface, $t^{\prime}=120 L / U$, and $S c=20,500$. In the horizontal directions periodic boundary conditions were employed and at the bottom symmetry boundary conditions $(\partial c / \partial z=0)$ were used.

The interfacial mass transfer simulation was carried out on the Supermuc cluster at LRZ in Munich using $\approx 18 \cdot 10^{6}$ CPUh and employing 20,992 cores.

\section{Results}

\subsection{Characteristics of flow field}

The interfacial mass transfer simulation was started using a fully developed turbulent flow field. This initial turbulent flow field was generated by running the simulation with- 


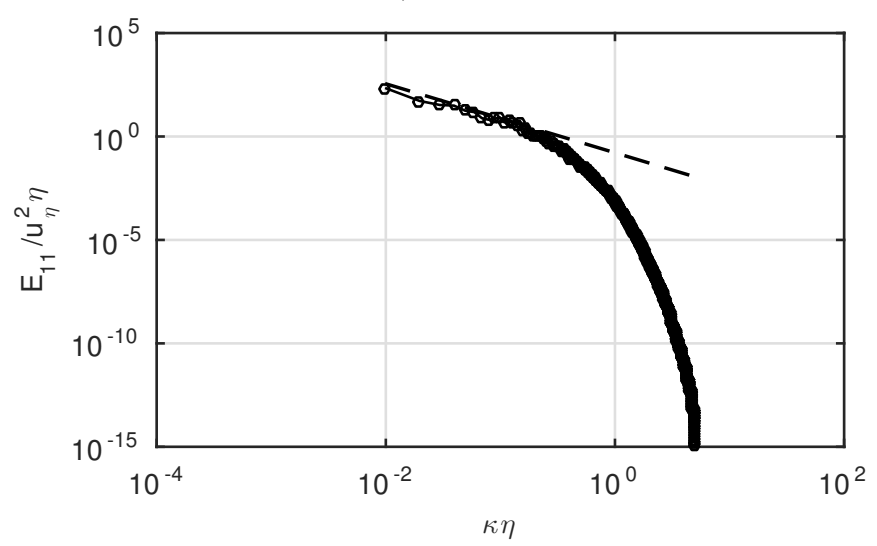

FiguRE 2. The mean energy spectrum normalised by the Kolmogorov velocity $u_{\eta}$ and length $\eta$ scales. The dashed line indicates the $-5 / 3$ slope.

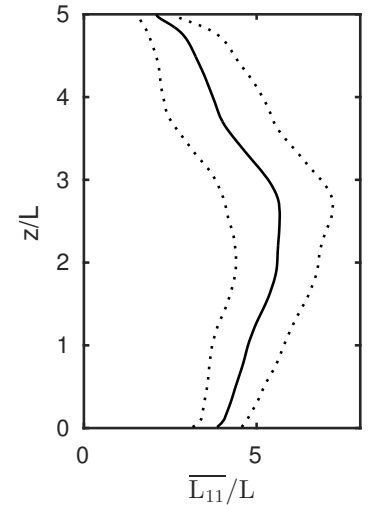

(a)

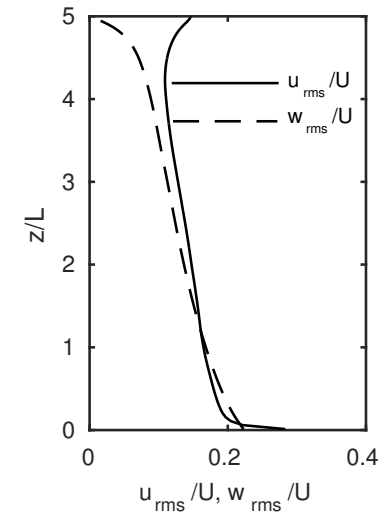

(b)

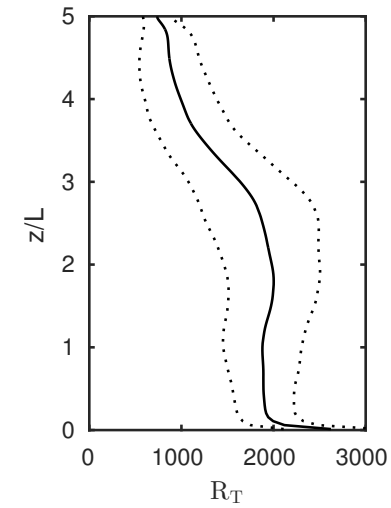

(c)

Figure 3. Flow statistics, ensemble averaged over $103 \mathrm{~L} / U$. (a) turbulent integral length scale $L_{11}$, (b) root-mean-square (rms) of horizontal $u$ and vertical $w$ velocity fluctuations, (c) turbulent Reynolds number. The dotted lines in (a) and (c) correspond to $\overline{L_{11}}(z / L) \pm \sigma_{L}$ and $R_{T}(z / L) \pm \sigma_{R}$, where $\sigma_{L}$ and $\sigma_{R}$ are the standard deviations of $L_{11}$ and $R_{T}$, respectively.

out calculating mass transfer. After a period of about $50 \mathrm{~L} / \mathrm{U}$, the turbulence in the DNS domain (upper box in figure 1) was found to be fully developed, as verified by the statistically steady behaviour of the turbulent energy spectrum shown in figure 2 . The mean energy spectrum, which was obtained by averaging the instantaneous energy spectrum at $z=2.75 L$ over the interval $50 L / U \leqslant t \leqslant 100 L / U$, clearly showed the existence of an inertial subrange with a $\kappa^{-5 / 3}$ behaviour, similar to Flores et al. (2017), as well as a broad dissipative range where $\kappa>\eta$. The same features were found in all spectra extracted at various other $z$ locations, indicating that the turbulent flow is very well resolved. Note that in the above $\kappa$ is the wavenumber and $\eta$ is the Kolmogorov length scale. Subsequently, at $t=100 L / U$ the full simulation was started by activating mass transfer.

The flow statistics shown in figure 3 were obtained by ensemble averaging the flow properties over the interval $50 L / U \leqslant t \leqslant 153 L / U$. By combining time averaging with 
averaging in the large homogeneous horizontal directions, the size of this relatively short interval was deemed to be sufficient to obtain good quality statistics. Figure 3a shows the variation in $z$ of the time-averaged longitudinal integral length scale $\overline{L_{11}}(-$ denotes averaging over a period in time), with

$$
L_{11}(z)=\int_{0}^{L_{x} / 2} R_{11}(r, z) \mathrm{d} r,
$$

where the longitudinal two-point correlation $R_{11}$ of the horizontal velocity $u$ is defined by

$$
R_{11}(r, z)=\frac{\int_{x=0}^{L_{x} / 2} \int_{y=0}^{L_{y}} u(x, y, z) u(x+r, y, z) \mathrm{d} y \mathrm{~d} x}{\int_{x=0}^{L_{x} / 2} \int_{y=0}^{L_{y}} u^{2}(x, y, z) \mathrm{d} y \mathrm{~d} x},
$$

in which $L_{x} \times L_{y}$ is the size of the horizontal plane. As in the grid-stirred experiments of e.g. Hopfinger \& Toly (1976); Herlina \& Jirka (2008), the integral length scale in most of the lower part of the computational domain was found to increase lineraly with increasing distance from the turbulent source. Furthermore, it can be seen that the characteristic integral length scale $L_{\infty}$, defined by

$$
L_{\infty}=\overline{L_{11}}\left(z_{\infty}\right)
$$

in which $z_{\infty}$ is the location where $\overline{L_{11}}$ is maximum, is about $5.67 \mathrm{~L}$. The characteristic integral length scale $L_{\infty}$ identifies the size of the largest eddies in the turbulent flow, which can only exist undisturbed at distances larger than one- $L_{\infty}$ from the interface. Closer to the interface, inside the so-called surface-influenced layer, the turbulence starts to lose its isotropy (cf. e.g. Hunt \& Graham 1978; Perot \& Moin 1995). Even though the depth of our DNS domain is somewhat smaller than $5.67 L$, a reasonable isotropy $\left(\left|1-w_{r m s} / u_{r m s}\right|<0.1\right)$ in most of the lower region $(z / L<2.5)$ is obtained (cf. figure 3b). In agreement with (e.g. Perot \& Moin 1995; Walker et al. 1996), the figure also shows an initial gradual decay in both horizontal and vertical fluctuations as the turbulence diffuses upwards. Close to the surface, a significant increase in decay rate of the vertical fluctuations $w_{r m s}$ is observed, which in turn induces an increase in the horizontal fluctuations $u_{r m s}$ due to the redistribution of the turbulent kinetic energy. A more detailed discussion on this for free-slip and/or no-slip boundary conditions can be found in e.g. Perot \& Moin (1995); Walker et al. (1996); Magnaudet (2003); Bodart et al. (2010); McCorquodale \& Munro (2017).

Typically, the turbulent Reynolds number for grid-stirred turbulence is defined by

$$
R_{T}=\frac{u_{\infty} \Lambda}{\nu}
$$

where $\Lambda=2 L_{\infty}$ (e.g. Brumley \& Jirka 1987; Hopfinger \& Toly 1976). This definition is also adopted here, where we used $u_{\infty}=u_{r m s}\left(z_{\infty}\right)$ for the characteristic velocity scale. The time-average of the corresponding local turbulent Reynolds number

$$
R_{T}(z)=u_{r m s} 2 \overline{L_{11}} / \nu,
$$

shown in figure $3 \mathrm{c}$, was found to be approximately constant $\left(R_{T}(z) \approx 1856\right)$ in most of the lower DNS domain. In general, the turbulent flow statistics were similar to the far-field statistics in the experiments of Brumley \& Jirka (1987); Herlina \& Jirka (2008) and the statistics obtained at low to moderate $R_{T}$ in HW14, where a more extended comparison with literature was presented. 


$\begin{array}{llll}\Delta t U / L & L_{\infty} / L & u_{\infty} / U & R_{T} \\ 50-153 & 5.67 & 0.1364 & 1856 \\ 126-153 & 4.54 & 0.1322 & 1440\end{array}$

TABLE 1. Mean flow parameters and the interval $\Delta t$ over which they are determined. Scalar statistics were only gathered over the smaller of the two intervals.

\subsection{Statistics of turbulent scalar transport}

As mentioned above, the scalar mass transfer calculations for $S c=20$ and 500 were activated at $t=100 \mathrm{~L} / U$ using the initial conditions given in (3.2). Owing to the high $R_{T}$, the transfer of saturated fluid from the surface to the bulk was relatively fast. This, together with the large horizontal extent of the computational domain, resulted in a speedy convergence of the normalised horizontally-averaged turbulent mass flux statistics. At $t=126 \mathrm{~L} / U$ time-units, the total mass flux near the interface $(0 \leqslant \zeta \leqslant 10 \delta)$ was found to be independent of $z$. Scalar transport statistics were subsequently gathered in this quasi-steady regime from $t=126 \mathrm{~L} / U$ until $153 \mathrm{~L} / U$.

As illustrated by the dotted lines in figure $3 \mathrm{a}$, the integral length scale of the turbulence $L_{11}$ varied significantly in time. Hence, it is expected that $L_{\infty}$ (and consequently $R_{T}$ ) - when calculated using a moving average over a relatively small time window- will also become time dependent. Using this we obtained a significant range of turbulent Reynolds numbers for the verification of the small-eddy model (cf. §4.4). Note that for the smaller time windows $L_{\infty}$ is the local maximum of $L_{11}$ nearest to the location where $L_{\infty}$ was obtained for the entire interval. The dependence of $L_{\infty}$ and $R_{T}$ on the size of the time interval can also be seen in table 1 , where when averaging over the entire interval $50 L / U-153 L / U, L_{\infty}=5.67 L$ and $R_{T}=1856$. On the other hand, by averaging over the smaller interval, values for $L_{\infty}$ and $R_{T}$ of $4.54 L$ and 1440 , respectively, were obtained.

The resulting scalar statistics can be seen in figure 4 . The vertical axes show the distance to the surface $\zeta$ normalised by the mean concentration boundary layer thickness $\delta$. The latter is defined by the distance to the surface where

$$
c_{r m s}=\sqrt{\overline{\left\langle c^{2}\right\rangle-\langle c\rangle^{2}}}
$$

is maximum. Note that $\langle\cdot\rangle$ denotes averaging in the homogeneous (horizontal) directions. Similarly to the lower $R_{T}$ cases in HW14, the horizontally-averaged boundary layer thickness $\delta$ was found to scale with $S c^{-1 / 2}$ as illustrated for $t=140 L / U$ in figure 5 (cf. further discussion in $\S 4.4)$.

Figure 4a shows the mean concentration profiles near the surface, normalised by calculating $\left(\overline{\langle c\rangle}-\overline{\left\langle c_{b}\right\rangle}\right) /\left(c_{s}-\overline{\left\langle c_{b}\right\rangle}\right)$. Immediately underneath the surface all profiles are approximately linear, which is a clear indication that molecular diffusion dominates gas transfer. It can also be seen that in that region the normalised profiles all collapse. For similar $S c$, deeper down in the bulk - due to the more effective vertical mixing - a slightly increased concentration at higher $R_{T}$ is observed. This is in agreement with the trend found for lower $R_{T}$ in HW14 and the experiments of Herlina \& Jirka (2008).

The normalised concentration fluctuations $c_{r m s} /\left(c_{s}-\overline{\left\langle c_{b}\right\rangle}\right)$, shown in figure $4 \mathrm{~b}$, can be seen to grow rapidly from zero at the surface to a local maximum, where approximately the horizontal turbulent exchange of saturated and unsaturated fluid is most intensive, before declining again to zero in the well-mixed region of the bulk. The peaks observed in the normalised $c_{r m s}$ (figure $4 \mathrm{~b}$ ) were found to be approximately 0.3 in all cases, 


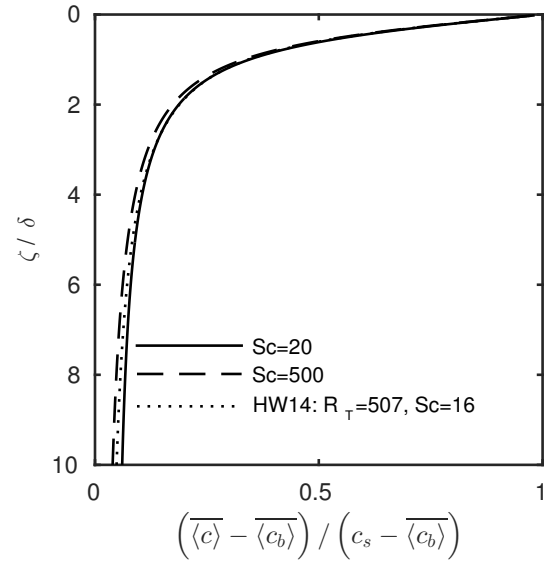

(a)

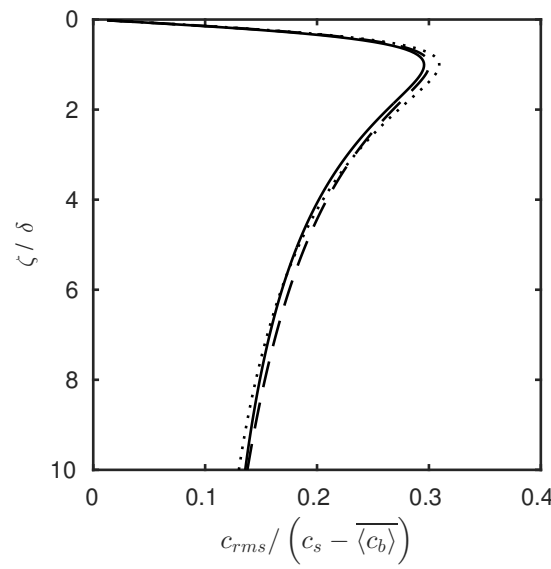

(b)

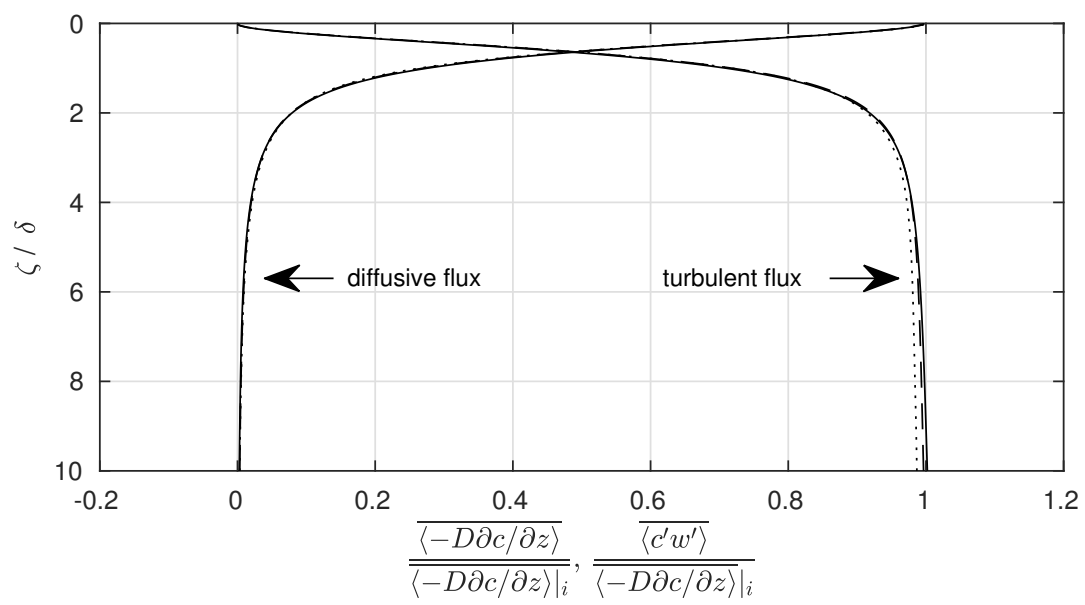

(c)

FIGURE 4. Scalar statistics: (a) normalised mean concentration profiles, (b) normalised rms profiles, and (c) normalised diffusive and turbulent scalar fluxes.

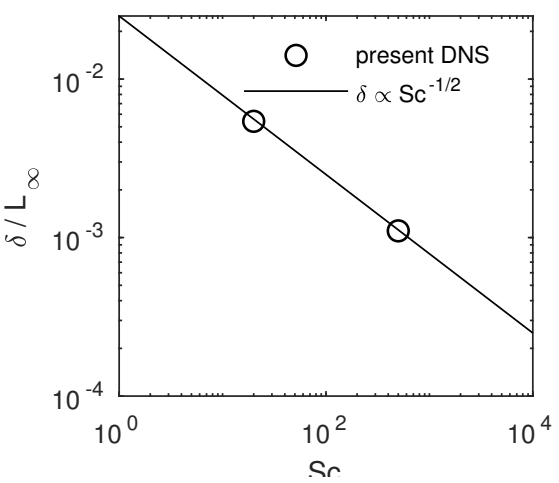

FigURE 5. Influence of $S c$ on the horizontally-averaged boundary layer thickness at $t=140 L / U$. 
which is typical for numerical simulations with free-slip surface boundary conditions (e.g. Magnaudet \& Calmet 2006) and is also found in some experiments (e.g. Atmane \& George 2002). Some other experiments, however, show $c_{r m s}$ peaks as low as 0.1 to 0.2 (e.g. Chu \& Jirka 1992; Herlina \& Jirka 2008). These relatively low peaks could be a consequence of a significant surface contamination as shown in the DNS studies of e.g. Khakpour et al. (2011); Wissink et al. (2017).

Figure $4 \mathrm{c}$ shows the mean diffusive $\overline{\langle-D \partial c / \partial z\rangle}$ and turbulent fluxes $\overline{\left\langle c^{\prime} w^{\prime}\right\rangle}$ normalised by the mean diffusive mass flux $\overline{\langle j\rangle}$ at the interface, where

$$
j=-\left.D \frac{\partial c}{\partial z}\right|_{i} .
$$

Present results for $R_{T} \approx 1440$ with $S c=20,500$ are shown together with the GS500 results from HW14 with $R_{T}=507$ and $S c=32$. In agreement with the theory, the diffusive fluxes are maximum at the surface and rapidly decrease to zero in the bulk, while simultaneously the turbulent fluxes increase from zero at the surface such that the sum of the mean diffusive and turbulent fluxes remains constant at all $z$-locations. The latter indicates that the simulation has run sufficiently long to achieve a quasisteady state. For all cases, the depth at which the normalised fluxes are in equilibrium is approximately the same $(\zeta \approx 0.65 \delta)$.

Note that even though the Schmidt numbers in the simulation differ significantly, all normalised scalar statistics shown in figure 4b,c nearly collapse. When applying the present normalisation to the lower $R_{T}$ cases in HW14, a similar data collapse is observed. This is illustrated by including the $R_{T}=507$ results (which are typical for $84 \leqslant R_{T} \leqslant$ $507)$ in the figure. Based on this, we can conclude that the normalised results are almost invariant with respect to both $S c$ and $R_{T}$.

\subsection{Role of large and small scales}

\subsubsection{Surface divergence}

Figure 6 depicts a comparison between the surface divergence $(\beta=-\partial w / \partial z)$ and the instantaneous mass transfer velocity for $S c=20$ at an arbitrarily chosen time $t=140 L / U$. The $\beta$ contours (figure 6a) clearly show the presence of convection cells corresponding to large structures of size $\sim L_{\infty}$ with positive surface divergence (upwelling of unsaturated fluid) separated by narrow regions of negative surface divergence (downwelling of saturated fluid). Compared to the surface divergence plot, the footprints of fine-scale turbulent structures can be more clearly identified in the corresponding instantaneous $K_{L}$ contour plot (for a more detailed comparison see figure 11). An important reason for this discrepancy is the much higher diffusivity of the fluid (affecting $\beta$ ) compared to the scalar (affecting $K_{L}$ ). An increase in $R_{T}$ leads to an increase in fine scale turbulent structures at the surface. Because of the aforementioned difference in diffusivities these structures will remain clearly visible for much longer in the $K_{L}$ contours than in the $\beta$ contours. This has a negative effect on the resulting time-averaged spatial correlation $\rho\left(K_{L}, \beta\right)$ at high $R_{T}$, as can be seen in figure 7, where in the present high $R_{T}$ simulation the value of $\rho\left(K_{L}, \beta\right)=0.78$ was lower than the values $\rho\left(K_{L}, \beta\right)=0.89,0.81,0.81$ obtained from HW14 for $R_{T}=84,195,507$, respectively. This reduction of $\rho\left(K_{L}, \beta\right)$ with $R_{T}$ also impacts the applicability of the surface divergence model at high $R_{T}$. This is in agreement with the findings of Turney \& Banerjee (2013) who observed a break down of the surface divergence model in the presence of small time scales. This will be further investigated below. 


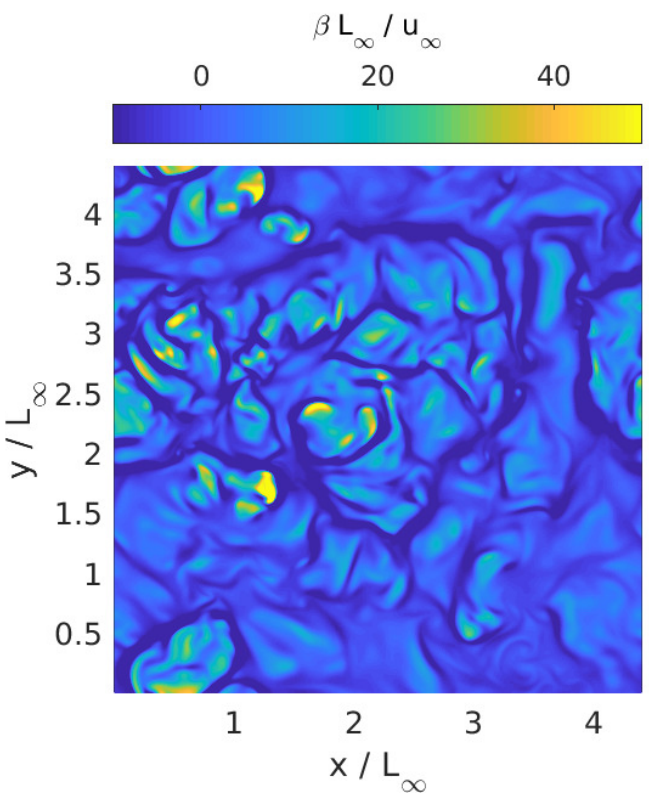

(a)

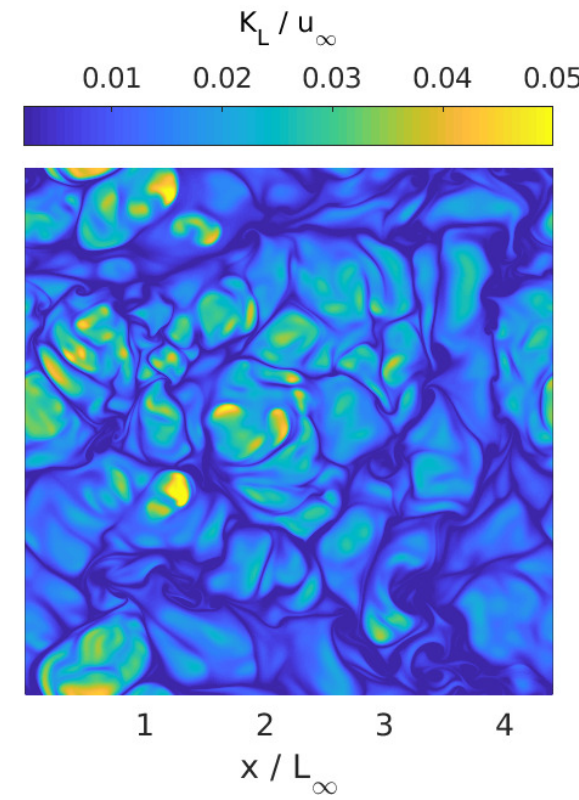

(b)

Figure 6. Snapshots at $t=140 L / U$ of (a) surface divergence $\beta$ and (b) $K_{L}$ for $S c=20$.

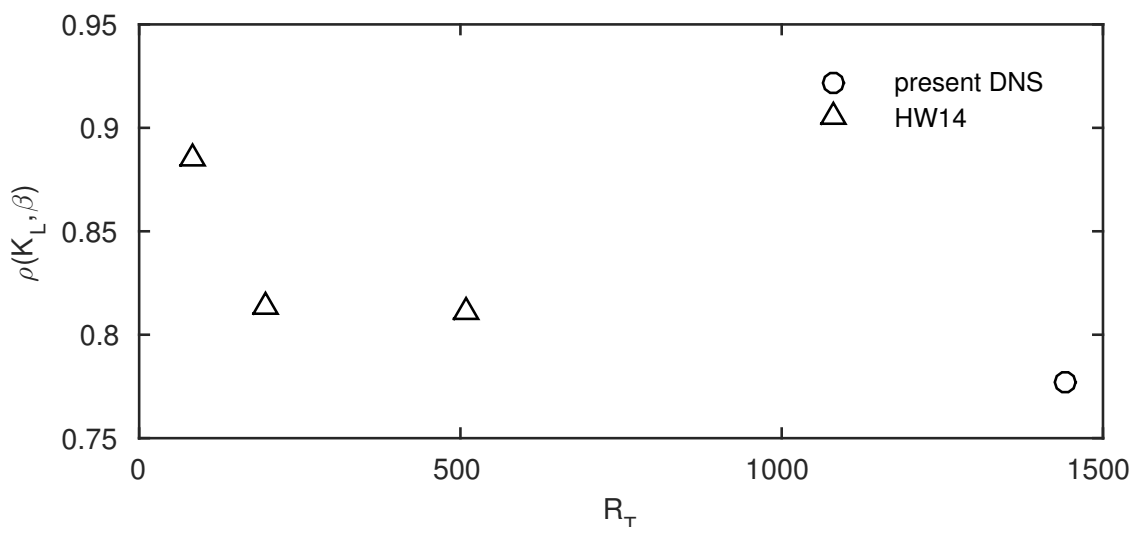

FIgURE 7 . Time-averaged spatial correlation of the transfer velocity $K_{L}$ with the surface divergence $\beta$ for various $R_{T}$.

\subsubsection{Vortical structures}

In figure 8 , vortical structures in part of the computational domains are identified using the $\lambda_{2}$ criterion of Jeong \& Hussain (1995). To allow a direct comparison of the structures obtained in the present DNS and GS500 (HW14), the $\lambda_{2}$ eigenvalues were normalised using the relevant characteristic turbulent length $\left(L_{\infty}\right)$ and velocity $\left(u_{\infty}\right)$ scales. Figures $8 \mathrm{a}$, b show that, because of the larger $R_{T}$, significantly more vortical structures are found in the present simulation than in the GS500 simulation, while the thickness of the structures - which depends on the Kolmogorov length scale - is smaller. The lack of vortical structures, as observed in large areas of the GS500 simulation, indicates that the 


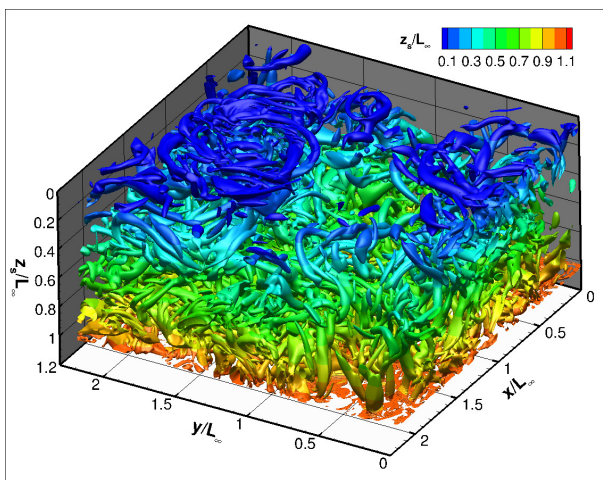

(a)

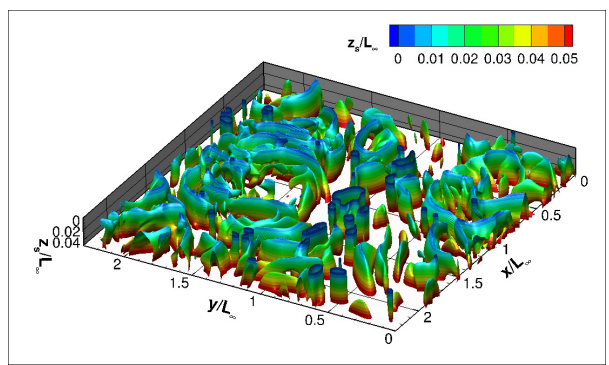

(c)

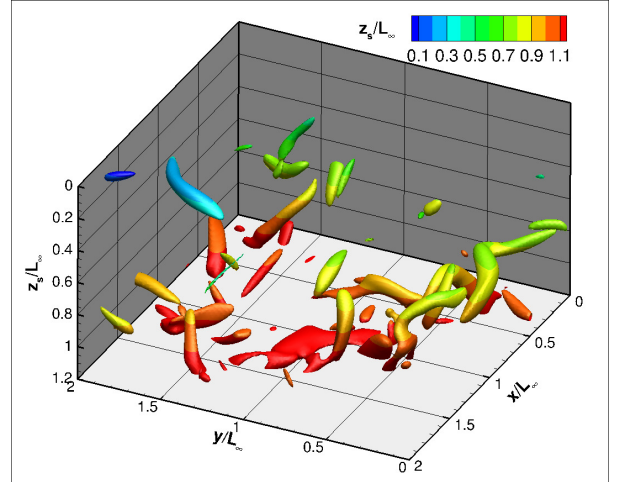

(b)

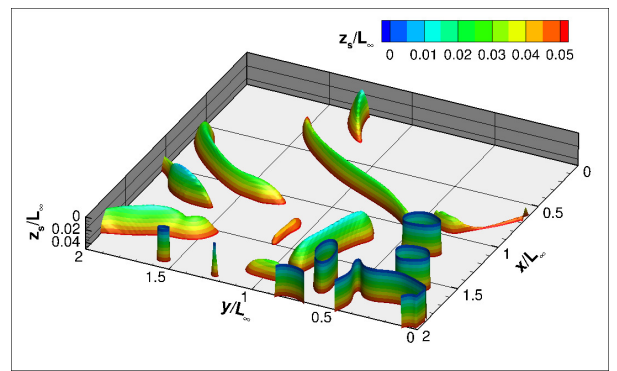

(d)

FiguRE 8. Snapshots of vortical structures identified using $\lambda_{2}=-200$ (upper panes) and $\lambda_{2}=-5$ (lower panes), normalised by $L_{\infty}$ and $u_{\infty}: \mathrm{a}$ ), c) present DNS at $t=146.6 L / U$ and b), d) case GS500 from HW14 $\left(R_{T}=507\right)$.

instantaneous turbulence is not uniformly distributed. Consequently, it is to be expected that the instantaneous mass transfer velocity will also not be uniformly distributed but will be elevated in regions with increased turbulence.

To further explore what actually happens close to the surface, in figures $8 \mathrm{c}, \mathrm{d}$ the vortical structures in the region $0 \leqslant \zeta / L_{\infty} \leqslant 0.04$ are visualised using $\lambda_{2}=-5$. Again, the number and size of the vortical structures in the present simulation differ significantly from the ones in GS500. As explained below, because of the free-slip boundary condition at the top, the axes of the structures close to the surface are either parallel or orthogonal to the interface. Figure 9 shows the Kolmogorov length scale $\eta$ for the present simulation as a function of the distance to the surface. Between $\zeta / L_{\infty} \approx 1$ (near the bottom) and $\zeta / L_{\infty} \approx 0.05, \eta$ was found to increase as the turbulence diffusing from below dissipates. As seen in figures $8 \mathrm{a}, \mathrm{c}$ and $3 \mathrm{~b}$, when further approaching the surface, the flow rapidly becomes more and more two-dimensional. In upwelling regions, the increase in horizontal velocities towards the surface stretches initially "randomly" orientated vortical structures and aligns them horizontally. Because of the large size of these upwelling regions (compared to the downwelling regions), this horizontal alignment is very common and causes vortical structures (on average) to become thinner towards the surface. As the diameter of the vortical structures scales with $\eta$ (Jimenez et al. 1993), the thinning mentioned above explains the decrease in $\eta$ observed in figure 9 between $\zeta / L_{\infty} \approx 0.05$ and 0.008 . Closer to the surface, the horizontal velocities become constant due to the free-slip boundary condition. As a result, the horizontal stretching no longer increases, which is an expla- 


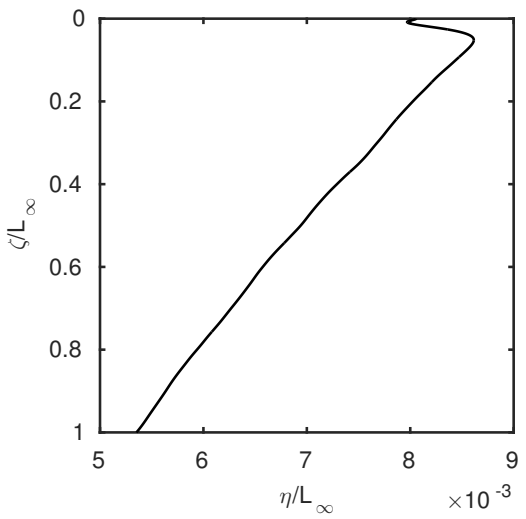

(a)

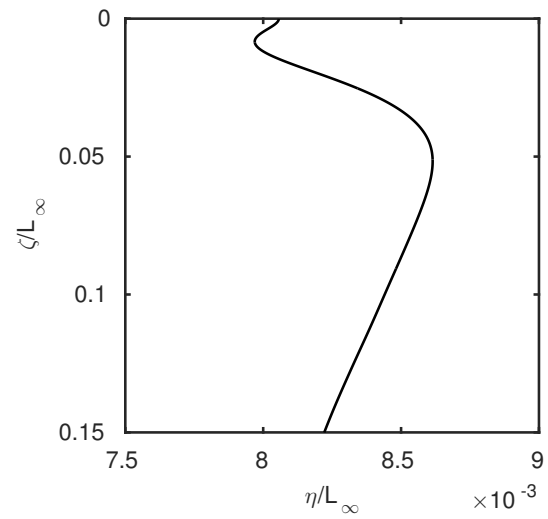

(b)

Figure 9. a) Variation of the mean Kolmogorov length scale with depth. b) Detailed view of (a) immediately beneath the surface.

nation of the slight increase in $\eta$ observed between $\approx 0.008$ and 0 . In the relatively small downwelling dominated regions, the downward velocity increases with distance from the surface thereby stretching and strengthening the local surface-attached (surface-normal) vortical structures (SAVS). Note that the SAVS observed in the downwelling regions are seeded by relatively weak surface-normal vorticity transported upwards in the upwelling regions, where they attach to the surface and are subsequently moved towards the downwelling regions.

While the surface-attached vortical structures promote mixing in the horizontal direction, the structures with axes parallel to the interface promote the exchange of fluid between the upper bulk and the surface. Their relation with mass transfer is studied in figure $10 \mathrm{a}$, by combining contours of $K_{L}$ with a cross-section of the vortical structures from figure $8 \mathrm{c}$ at $\zeta / L_{\infty}=0.01$. The horizontally-aligned slender vortical structures can be found near the edges of areas with high $K_{L}$, adjacent to locations where saturated fluid is transported downwards. The SAVS, on the other hand, are generally located in areas with relatively low $K_{L}$. To distinguish the SAVS from the horizontally-aligned vortical structures, in figure 10b, the $\lambda_{2}$ isolines are combined with contours of the surface normal vorticity component $\omega_{z}$. In contrast to the horizontally-aligned vortices, SAVS are marked by elevated levels of $\omega_{z}$ in their entire interior.

Figure 11 shows a detailed view of the $\beta$ and $K_{L}$ contours from figure 6 combined with isolines of relatively high-intensity surface-normal vorticity $\left|\omega_{z}\right|$. As mentioned above, because of the stretching of SAVS, it is expected to only see such isolines in downwelling areas. When this downwelling is very strong, SAVS undergo significant stretching and quickly become exceedingly thin leading to their dissipation within a relatively short time. SAVS in areas of very weak downwelling (as seen in figure 11a), tend to survive for a long time in the absence of large-scale turbulent motion. Because of the extended exposure time at the surface the fluid inside these structures has become highly saturated due to diffusive mass transfer, as indicated by the local low $K_{L}$ values in figure $11 \mathrm{~b}$. While the correlation of these SAVS with low $K_{L}$ values is very good, the correlation with low $\beta$ values is rather poor. This indicates that the local correlation $\rho\left(\beta, K_{L}\right)$ will be rather small. Note that the above observations are generally confirmed in all recorded snapshots. 


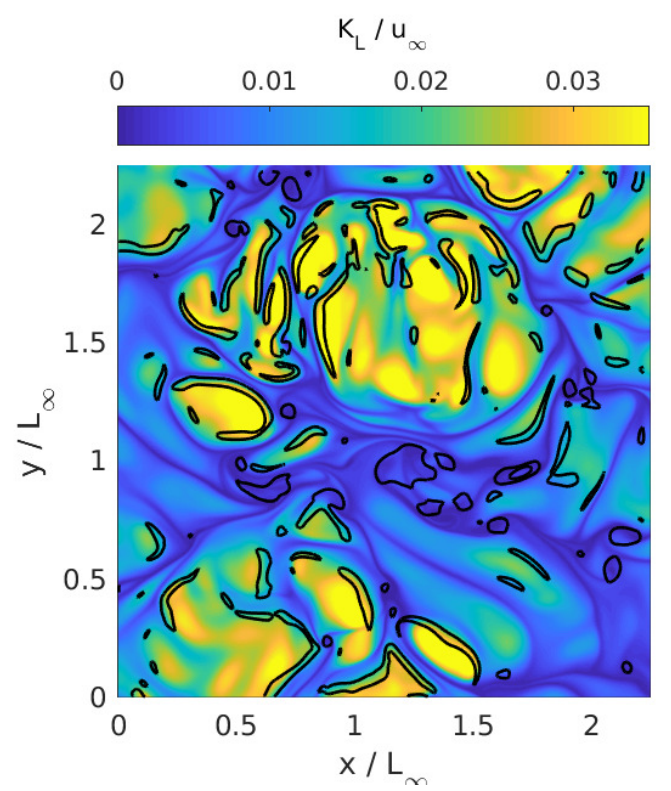

(a)

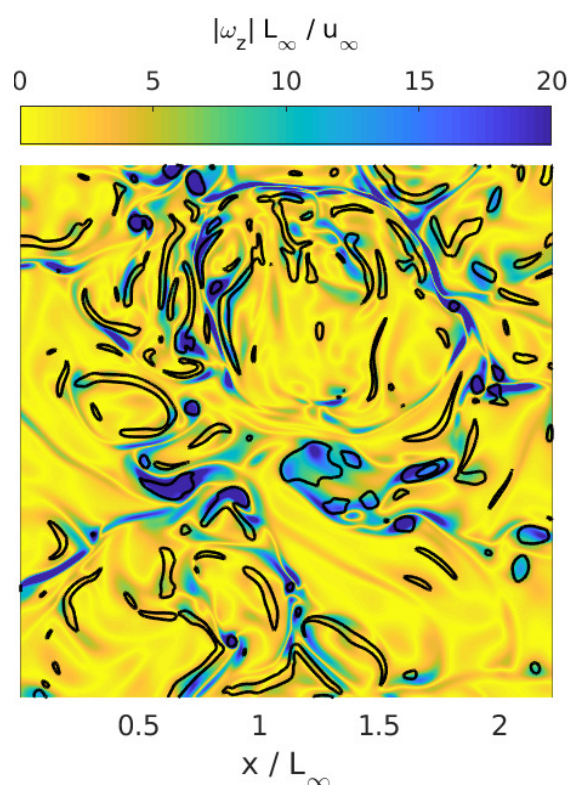

(b)

FiguRE 10. Isolines of $\lambda_{2}=-5$ at $\zeta / L_{\infty}=0.01$ and $t=146.6 \mathrm{~L} / U$ with a) contours of $K_{L}$ for $S c=20$ and b) contours of $\left|\omega_{z}\right|$. Note that $\lambda_{2}, K_{L}$ and $\omega_{z}$ were normalised using $L_{\infty}$ and $u_{\infty}$.

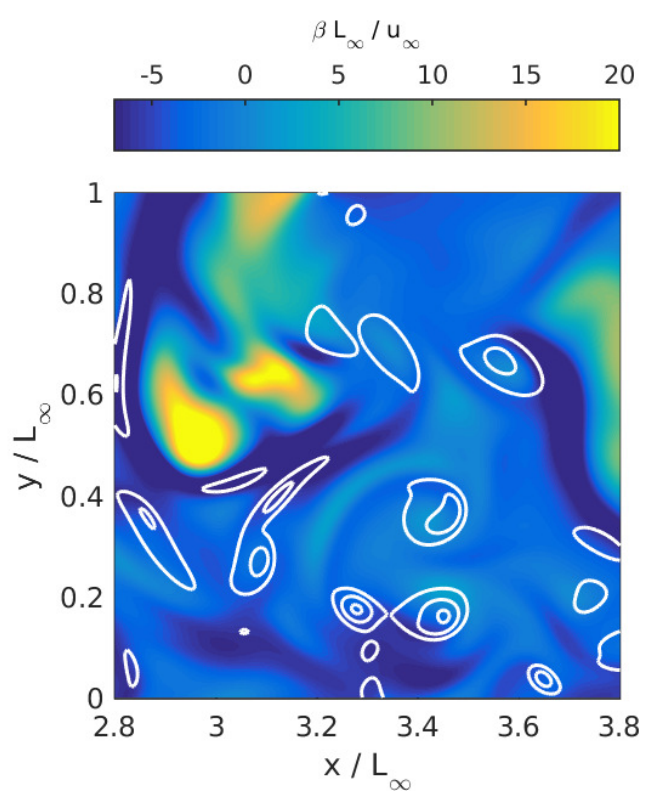

(a)
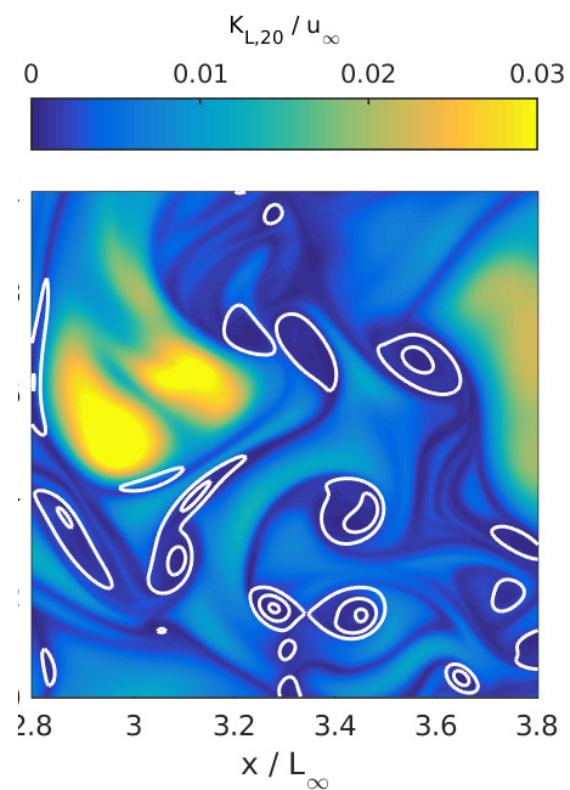

(b)

FIgURE 11. Zoomed view of figure 6 combined with isolines of surface-normal vorticity magnitude at $\left|\omega_{z}\right| \frac{L_{\infty}}{u_{\infty}}=14,34,51$ and 68. a) Contours of $\beta$ and b) contours of $K_{L}$. 
The dynamics of vortical structures in open-channel flow and their influence on interfacial mass transfer has been discussed by e.g. Pan \& Banerjee (1995); Nagaosa (1999); Nagaosa \& Handler (2003). Pan \& Banerjee (1995) discovered the existence of relatively large ring-like vortices at the surface of open channel flow that are associated with vertical mixing. In the interior of the ring unsaturated fluid from the bulk is transported towards the surface (splat) while immediately outside of the ring saturated fluid is transported down into the bulk (antisplat). Nagaosa \& Handler (2003) found that the ring-like vortical structures originate from near the bottom of the channel and start their life as hairpin vortices. In our case, in the absence of bottom-shear and associated vortical structures like hairpin vortices, the vortical dynamics of the turbulent flow changes significantly. Individual upwellings are usually relatively small but tend to cluster together into larger areas separated by narrow regions with strong downwelling. The topology of the clusters is very complex and rapidly changes in time. As discussed above (see also figure 10), the vortices parallel to the surface, that are responsible for vertical mixing, tend to lie along the edges of upwelling regions and sometimes resemble parts of ring-like vortices, similar to the ones previously observed by e.g. Nagaosa \& Handler (2003). As mentioned earlier, the SAVS only promote horizontal mixing and hence do not directly contribute to the turbulent vertical mass transfer, which is in agreement with the findings of Nagaosa (1999).

\subsubsection{Spectra of turbulent mass flux}

As illustrated above, the mass transfer is directly related to the presence of vortical structures immediately underneath the surface. With increasing $R_{T}$ the quantity of vortical structures increases, while their diameter typically decreases. To further investigate the relative importance of large and small scale structures for vertical mass transfer, time-averaged spectra of the turbulent mass flux $c^{\prime} w^{\prime}$ are plotted as a function of the normalised wavenumber $\kappa L_{\infty}$ (cf. figure 12). The present results, obtained at $R_{T}=1440$, are compared to the results of GS500 at $R_{T}=507$ and GS80 at $R_{T}=84$ (see HW14).

Figure 12a shows the cospectra premultiplied by the wavenumber $\kappa$. Compared to the present simulation at $R_{T}=1440$, the energy-containing range at $R_{T}=507$ moves to smaller values of $\kappa L_{\infty}$. At $R_{T}=84$ only a relatively narrow band of energy-containing scales was observed, which coincides with the largest energy-containing scales observed at $R_{T}=507$. Hence, it can be concluded that at low $R_{T}$ large scales dominate the turbulent mass flux, while at high $R_{T}$ the contribution of the small scales becomes more important, supporting the two-regime mass-transfer concept of Theofanous et al. (1976). Near the critical $R_{T}$ both small and large scales were found to contribute significantly to the overall turbulent mass transfer.

To further illustrate the contribution of the different scales to the mass transfer, the cumulative cospectra of $c^{\prime} w^{\prime}$ are shown in figure $12 \mathrm{~b}, \mathrm{c}$ at $\zeta=5 \delta$ and $\delta$, respectively. It can be seen that with increasing $R_{T}$ the smaller scales become more and more important for the vertical turbulent mass transport. For instance, at $\zeta=5 \delta$, the contribution of relatively large scales with wavenumbers $\kappa L_{\infty} \leqslant 10$ to the total energy of the turbulent flux decreases from $70 \%$ for $R_{T}=84$ to $55 \%$ for $R_{T}=507$ and finally to $34 \%$ for $R_{T}=1440$. Higher up in the boundary layer, at $\zeta=\delta$, the presence of the free-slip surface causes the flow to become increasingly two-dimensional. This is evidenced by the change in orientation of the vortical structures when approaching the surface as mentioned above (cf. figure 8a,c). The inverse energy cascade, that is typical for 2D turbulence, explains the increased importance of the larger scales to the vertical mass transfer when approaching the surface (cf. figure 12c). 


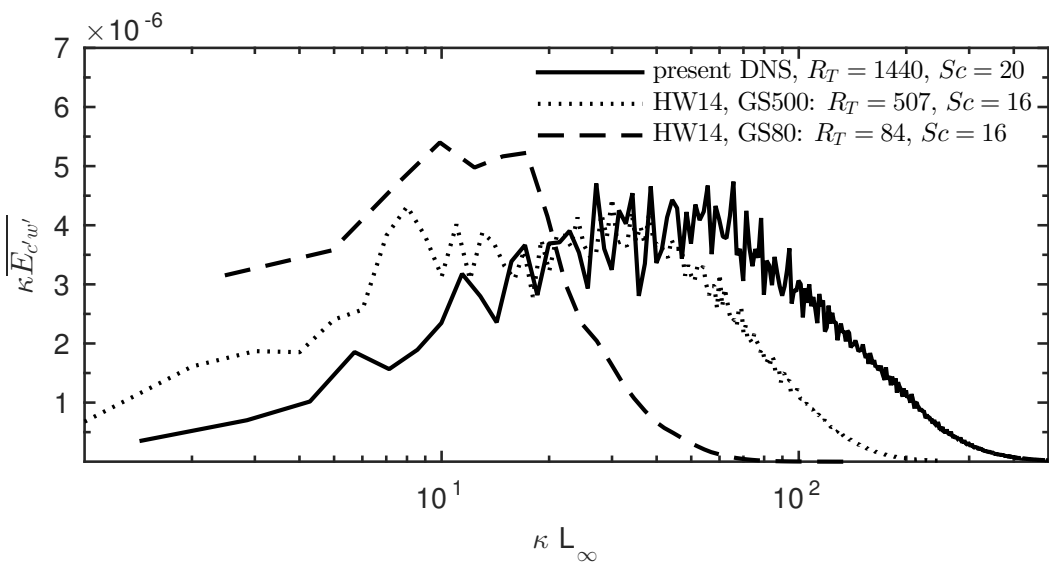

(a)

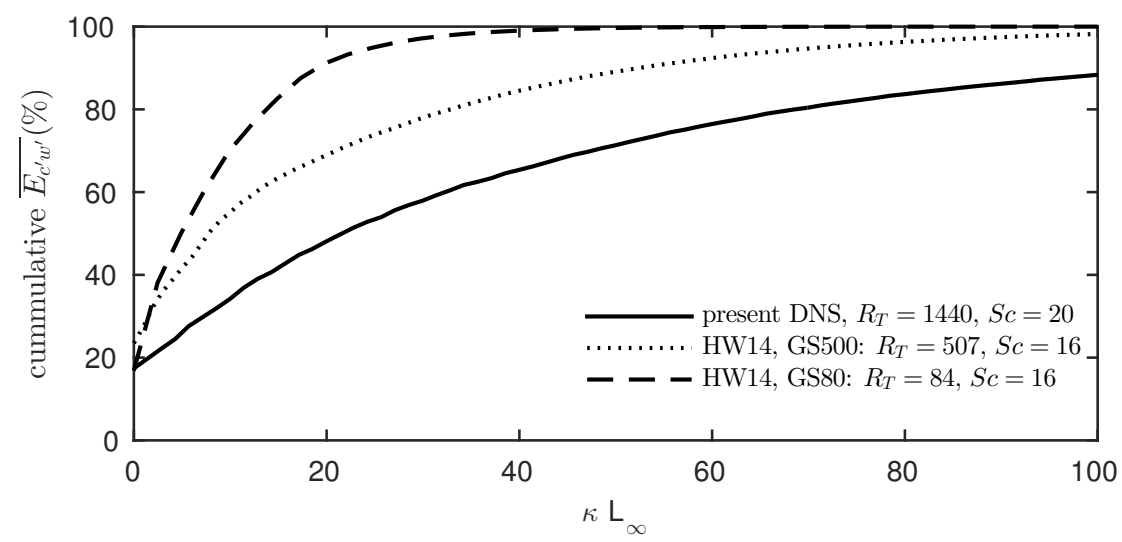

(b)

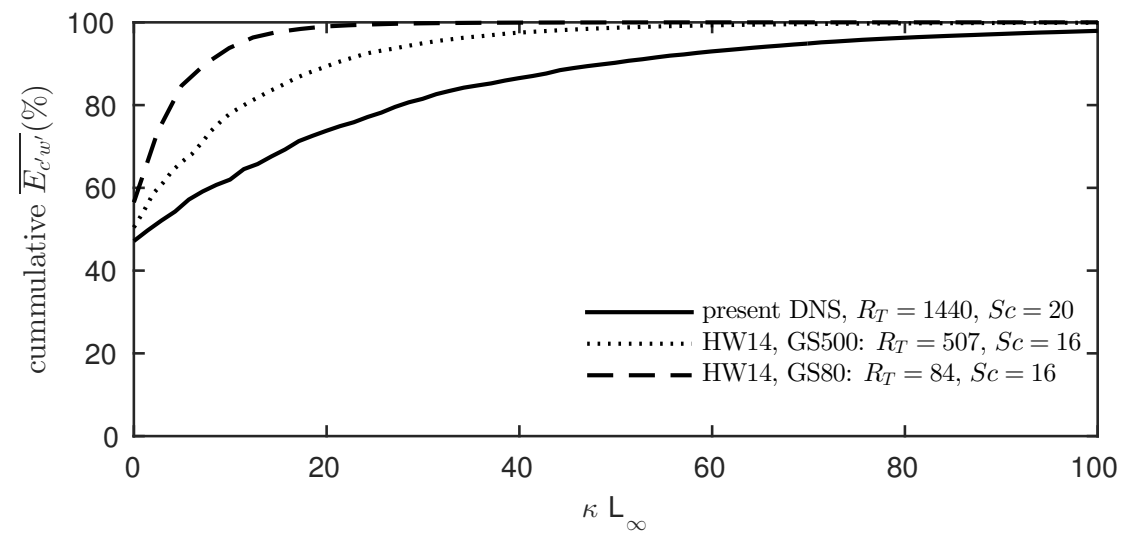

(c)

Figure 12. a) Premultiplied cospectra of turbulent mass flux $c^{\prime} w^{\prime}$ at $\zeta=5 \delta$. Cumulative cospectra of $c^{\prime} w^{\prime}$ at b) $\zeta=5 \delta$ and c) $\zeta=\delta$. 


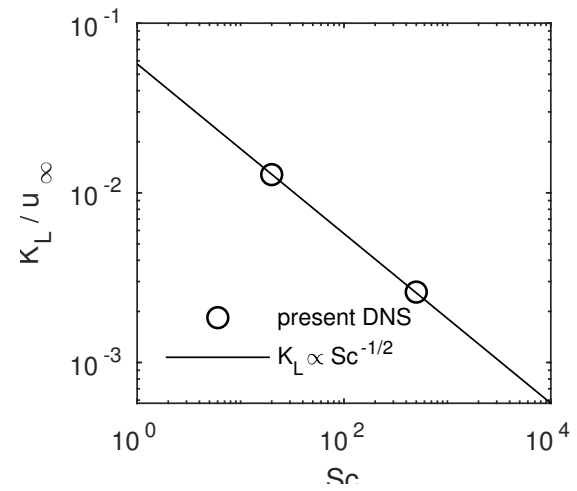

Figure 13. Influence of $S c$ on $K_{L}$. Data shown are from the snapshot at $t=140 L / U$.

\subsection{Scaling of transfer velocity with $S c$ and $R_{T}$}

In agreement with experiments of e.g. Chu \& Jirka (1992); Atmane \& George (2002); Herlina \& Jirka (2008), in the HW14 simulations it was found that the concentration boundary layer thickness $\delta$ depends on the level of isotropic turbulence diffusing from below. This turbulence promotes the interchange of unsaturated fluid from the bulk with saturated fluid from the surface. For $S c$ is constant, the boundary layer thickness (normalised by $L_{\infty}$ ) decreases with increasing turbulence level, resulting in a steeper concentration gradient at the surface and therefore an increased normalised mass transfer velocity. On the other hand, for $R_{T}$ is constant it was shown that $\delta$ scales with $S c^{-1 / 2}$ (cf. figure 5). The same scaling was found for $K_{L}$, as can be seen in figure 13 . This implies that for constant $R_{T}$ and free-slip surface boundary conditions, the mass transfer velocity $K_{L}$ is proportional to $\delta$. Note that the same instantaneous scaling behaviour $K_{L} \propto S c^{-1 / 2}$ was consistently found for all $t \geqslant 126 L / U$. Also, this scaling is in agreement with the theoretical scaling found for free-slip surface conditions (e.g. Jähne \& Haussecker 1998; Ledwell 1984).

Free-slip boundary conditions are applied to represent a clean (surfactant-free) surface. Contaminated surfaces, on the other hand, can be modelled e.g. by using a no-slip boundary condition to represent some of the physics pertinent to severe contamination (Herlina \& Wissink 2016) or by explicitly solving the surfactant transport at the surface and modelling their influence on the near-surface velocity field (Shen et al. 2004; Khakpour et al. 2011; Wissink et al. 2017). As seen in Wissink et al. (2017), the scaling behaviour of the mass transfer velocity with the Schmidt number smoothly changes from $S c^{-1 / 2}$ for clean surfaces to $S c^{-2 / 3}$ for severely contaminated surfaces.

As mentioned above, the present high- $R_{T}$, high- $S c$ DNS study builds on our previous DNS of interfacial mass transfer driven by isotropic turbulence diffusing from below, see Herlina \& Wissink (2014, 2016); Wissink et al. (2017). Figure 14 shows the normalised mass transfer velocity as a function of the turbulent Reynolds number $R_{T}$,

$$
\frac{K_{L}}{u_{\infty} S c^{-n}}=c R_{T}^{-q}
$$

where $n=1 / 2$ and $n=2 / 3$ for the free-slip and no-slip surface boundary conditions, respectively, while $q$ depends on the size of the dominating turbulent scales, as discussed below. Note that after using $u_{\infty}$ and $\Lambda$ for nondimensionalisation, it can be seen that for $n=q=1 / 2(4.5)$ is equivalent to (1.3), while for $n=1 / 2$ and $q=1 / 4$ it is equivalent to 


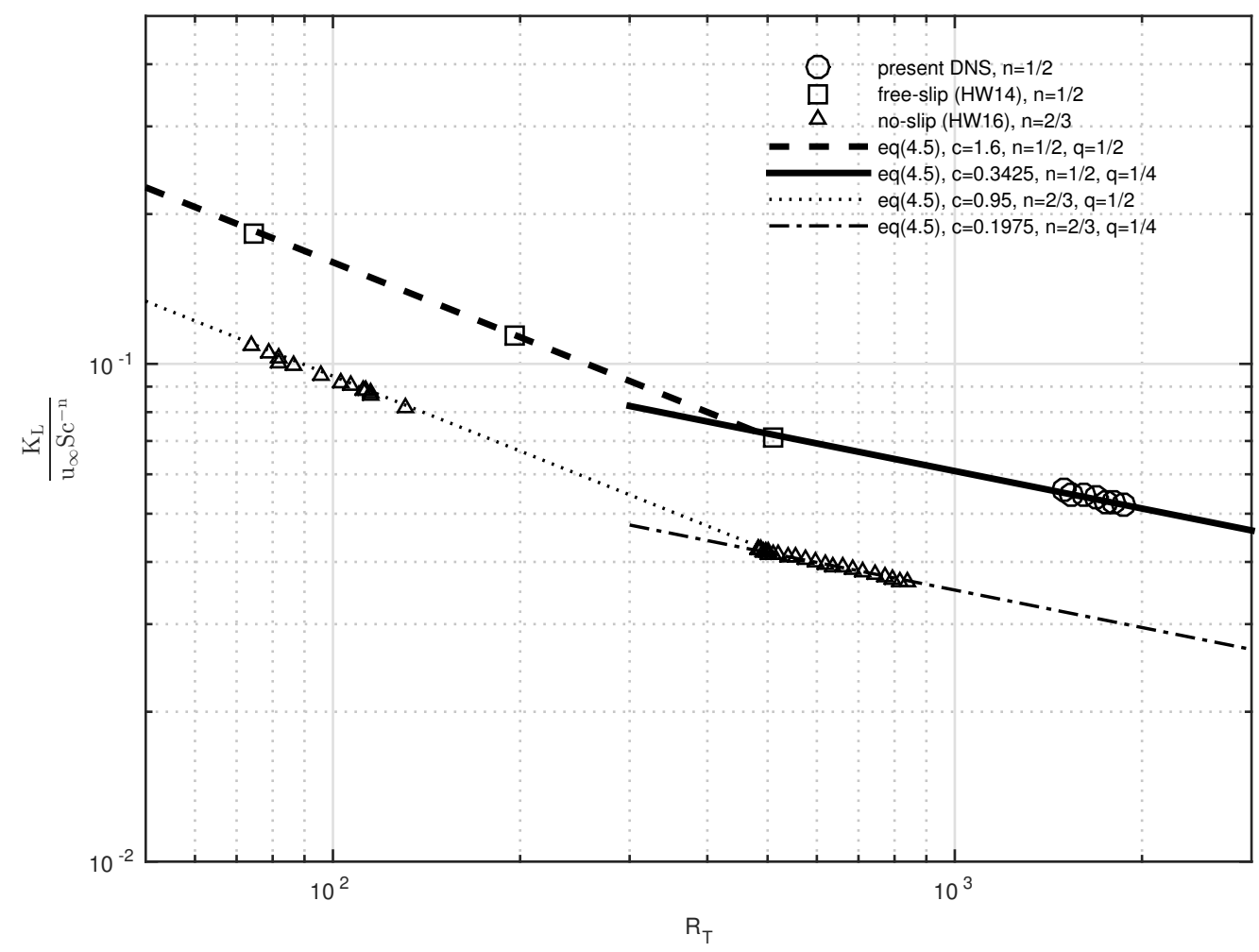

FiguRE 14. Variation of the normalised $K_{L}$ with $R_{T}$. For the free-slip boundary condition, the present DNS results are combined with results from HW14 obtained at low to moderate $R_{T}$ (the thick lines represent the theoretical scaling laws). In addition, results from HW16, using a no-slip boundary condition, are also shown (here thin lines represent the theoretical scaling laws).

(1.4), cf. Theofanous et al. (1976). The data shown in the figure originate from our previous (HW14) and present DNS of interfacial mass transfer driven by isotropic turbulence with free-slip surface boundary conditions. While HW14 only considered low to moderate $R_{T}$ values, our present DNS for the first time combines high $R_{T}$ turbulence $\left(R_{T} \approx 1440\right)$ with low diffusivity mass transfer ( $S c$ up to 500), which is typical for dissolved oxygen in water. In agreement with Theofanous (1984), two regimes can be identified in this figure. For turbulent Reynolds numbers smaller than $R_{T} \approx 500$, the HW14 numerical results show a scaling of the normalised mass transfer velocity with $R_{T}^{-1 / 2}$, which supports the large-eddy model of Fortescue \& Pearson (1967). For larger $R_{T}$, our present DNS results - obtained by combining horizontal averaging with ensemble averaging over time periods of $30 L / U$ - clearly show the presence of a different scaling law where $\frac{K_{L}}{u_{\infty} S c^{-1 / 2}} \propto R_{T}^{-1 / 4}$, which is in agreement with the small-eddy model of Banerjee et al. (1968); Lamont \& Scott (1970). The same $K_{L}$ dependency on $R_{T}^{-1 / 4}$ was reported in the experimental work of Herlina \& Jirka (2008). The data shown in the figure is complemented by results from our no-slip simulations (cf. HW16). As in the free-slip case, both the small and large eddy regimes can be clearly identified. It can be seen in Herlina \& Wissink (2016) that 
the fitted line through the free-slip numerical results for high $R_{T}$ also agrees well with the upper bound of the experimental findings of McKenna \& McGillis (2004) obtained using a clean surface. Note that, even though we expect a smooth transition between the two regimes, it was found that the line through the present free-slip DNS data also intersects the result obtained in HW14 at $R_{T}=507$. Further investigations would be needed to determine a detailed picture of the transitional regimes around $R_{T} \approx 500$ for both the free-slip and the no-slip case.

\section{Conclusions}

A large scale direct numerical simulation of interfacial mass transfer at moderate to high Schmidt numbers (up to $S c=500$ ) across a free-slip surface, driven by relatively high intensity isotropic turbulence $\left(R_{T} \approx 1440-1856\right)$, has been performed. The isotropic turbulence, generated in a concurrently running LES, was introduced at the bottom of the DNS domain. It was found that the missing subgrid scales in the LES energy spectrum established very rapidly as the turbulence diffused upwards in the DNS domain. Even though the depth of the computational domain was relatively small compared to the integral length scale, the statistics typical for shear-free turbulence near a free-slip surface were found to be consistent with previous data. For instance, the time-averaged turbulent Reynolds number was found to be approximately constant in most of the lower part of the DNS domain.

Previous numerical investigations of interfacial mass transfer driven by turbulence were mostly limited to low $R_{T}$ and/or low $S c$. As far as the authors are aware, this is the first DNS in which both the Kolmogorov scale of the relatively highly turbulent flow (significantly higher than the critical $R_{T} \approx 500$, above which the small eddy model is believed to be applicable for the estimation of the mass transfer velocity $K_{L}$ ) and the Batchelor scale, typical for the low-diffusivity mass transfer, are resolved. This was made possible by employing a dual-mesh approach in which the turbulent flow and the lower Schmidt number $(S c=20)$ scalar transport were resolved on the base mesh comprising $524 \times 10^{6}$ grid points, while a refined mesh of $65.5 \times 10^{9}$ grid points was used for the higher Schmidt number $(S c=500)$ scalar transport, which is typical for oxygen in water. The presence of an inertial subrange with a $\kappa^{-5 / 3}$ behaviour and a broad dissipative range in the energy spectra, as found at various $z$-locations, further confirms that the turbulent flow in the DNS is very well resolved.

Independent of $R_{T}$ and $S c$, the profiles of the mean concentration, the concentration fluctuation, the diffusive flux and the turbulent mass flux obtained in this simulation and our previous simulations (HW14) were found to nearly collapse when applying suitable normalisations. In agreement with the theory, the sum of the diffusive and turbulent fluxes in the upper bulk was observed to be equal to the diffusive flux at the surface.

At constant $R_{T}$, the scaling of both $\delta$ and $K_{L}$ with $S c^{-1 / 2}$ was found to be accurately reproduced, indicating that $K_{L}$ varies linearly with $\delta$. At constant $S c$, however, with increasing $R_{T}, \delta / L_{\infty}$ is expected to become smaller resulting in a steeper gradient of the vertical concentration profile near the surface giving rise to an increase in $K_{L}$.

The instantaneous correlation of the surface divergence with the local $K_{L}$ at the surface was found to worsen with increasing $R_{T}$. As suggested by Turney \& Banerjee (2013), this can be attributed to the presence of small time scales in the highly turbulent flow. Compared to our previous simulation at a lower $R_{T}=507$, here the vortical structures were found to be smaller and far more numerous. As a result, the contribution of the smaller scales to the total turbulent mass flux was observed to be significantly larger than in the lower $R_{T}$ simulations. Also, close to the surface the turbulent intensity in the 
present simulation remains highly significant, while the axes of the vortical structures in both simulations either became aligned with or orthogonal to the surface. The latter is a consequence of the flat, free-slip boundary condition at the top forcing the turbulent flow to become increasingly two-dimensional. The structures that aligned with the surface were located at the boundaries of upwelling and downwelling regions and contributed to the vertical mixing of saturated and unsaturated fluid, which at the surface is characterised by relatively high levels of $K_{L}$. The orthogonal (surface-attached) structures, on the other hand, were mostly located in highly saturated areas and merely mixed already saturated fluid in the horizontal direction.

Previously, the existence of the small and large-eddy regimes in the presence of a no-slip surface boundary conditions was confirmed numerically in Herlina \& Wissink (2016). By ensemble averaging the data in the present simulation, we were able to obtain the mass transfer velocities for a range of $R_{T}$ values between approximately 1440 and 1856 . In line with the observations above, the importance of small scale turbulent structures was further confirmed by the scaling of the normalised mass transfer velocity $\frac{K_{L}}{u_{\infty} S c^{-1 / 2}}$ with $R_{T}^{-1 / 4}$. The latter corresponds to the small-eddy model of Banerjee et al. (1968); Lamont \& Scott (1970), which according to Theofanous (1984) is applicable for $R_{T}$ larger than $\approx 500$. Combining results from the present simulation with results from HW14, confirmed that for interfacial mass transfer driven by isotropic turbulence diffusing from below, the critical $R_{T}$ is indeed about 500. It is likely, however, that there will be a smooth transition between the large-eddy and the small-eddy model, which needs further investigation.

\section{Acknowledgements}

The authors would like to thank the German Research Foundation (DFG) for funding this project and the Gauss Centre for Supercomputing e.V. (www.gauss-centre.eu) for providing computing time on the GCS Supercomputer SuperMUC at Leibniz Supercomputing Centre (LRZ, www.lrz.de). Finally, the anonymous referees are acknowledged for their helpful comments. The data used in various plots can be found in http://www.ifh.kit.edu/dns_data/gas_transfer.

\section{REFERENCES}

Atmane, M. A. \& George, J. 2002 Gas transfer across a zero-shear surface: a local approach. Geoph Monog 127, Gas Transfer at Water Surfaces pp. 255-259.

Banerjee, S., Scott, D. S. \& Rhodes, E. 1968 Mass transfer to falling wavy liquid films in turbulent flow. Ind. Eng. Chem. Fund. 7 (1), 22-27.

Bodart, J., Cazalbou, J. B. \& Joly, L. 2010 Direct numerical simulation of unsheared turbulence diffusing towards a free-slip or no-slip surface. J. Turbul. 11, N48.

Brumley, B. H. \& JiRKA, G. H. 1987 Near-surface turbulence in a grid-stirred tank. J. Fluid Mech. 183, 235-263.

Brumley, B. H. \& JiRKA, G. H. 1988 Air-water transfer of slightly soluble gases: turbulence interfacial processes and conceptual models. Physicochem. Hydrodyn. 10 (3), 295-319.

Chu, C. R. \& JiRkA, G. H. 1992 Turbulent gas flux measurements below the air-water interface of a grid-stirred tank. Int. J. Heat Mass Tran. 35 (8), 1957-1968.

Danckwerts, P. V. 1951 Significance of liquid-film coefficients in gas absorption. Ind. Eng. Chem. 43 (6), 1460-1467.

Flores, O., Riley, J. J. \& Horner-Devine, A. R. 2017 On the dynamics of turbulence near a free surface. J. Fluid Mech. 821, 248265.

Fortescue, G. E. \& Pearson, J. R. 1967 On gas absorption into a turbulent liquid. Chem. Eng. Sci. 22 (9), 1163-1176.

Fredriksson, S. T., Arneborg, L., Nilsson, H., Zhang, Q. \& Handler, R.A. 2016 An 
evaluation of gas transfer velocity parameterizations during natural convection using DNS. J. Geophys R: Oceans 121 (2), 1400-1423.

Grötzbach, G. 1983 Spatial resolution requirements for direct numerical simulation of the Rayleigh-Bènard convection. J. Comp. Phys. 49, 241-264.

Handler, R. A., Saylor, J. R., Leighton, R. I. \& Rovelstad, A. L. 1999 Transport of a passive scalar at a shear-free boundary in fully turbulent open channel flow. Phys. Fluids $11(9), 2607-2625$.

Hasegawa, Y. \& Kasagi, N. 2008 Systematic analysis of high Schmidt number turbulent mass transfer across clean, contaminated and solid interfaces. Int. J. Heat Fluid Flow 29 (3), $765-773$

Herlina \& JiRKa, G. H. 2008 Experiments on gas transfer at the air-water interface induced by oscillating grid turbulence. J. Fluid Mech. 594, 183-208.

Herlina, H. \& Wissink, J.G. 2014 Direct numerical simulation of turbulent scalar transport across a flat surface. J. Fluid Mech. 744, 217-249.

Herlina, H. \& Wissink, J. G. 2016 Isotropic-turbulence-induced mass transfer across a severely contaminated water surface. J. Fluid Mech. 797, 665-682.

Hopfinger, EJ \& Toly, J-A 1976 Spatially decaying turbulence and its relation to mixing across density interfaces. J. Fluid Mech. 78 (1), 155-175.

Hunt, J. C. R. \& Graham, J. M. R. 1978 Free-stream turbulence near plane boundaries. J. Fluid Mech. 84, 209-235.

Jähne, B. \& Haussecker, H. 1998 Air-water gas exchange. Annu. Rev. Fluid Mech. 30, 443468.

Janzen, J. G., Herlina, H., Jirka, G.H., Schulz, H.E. \& Gulliver, J.S. 2010 Estimation of mass transfer velocity based on measured turbulence parameters. AIChE Journal $\mathbf{5 6}$ (8), 2005-2017.

JeOng, J. \& Hussain, F. 1995 On the identification of a vortex. J. Fluid Mech. 285, 69-94.

Jimenez, J., Wray, A.A., Saffman, P.G. \& Rogallo, R.S. 1993 The structure of intense vorticity in isotropic turbulence. J. Fluid Mech. 255, 65-90.

Khakpour, H.R., Shen, L. \& Yue, D.K.P. 2011 Transport of passive scalar in turbulent shear flow under a clean or surfactant-contaminated free surface. J. Fluid Mech. 670, 527-557.

Kubrak, B., Herlina, H., Greve, F. \& Wissink, J.G. 2013 Low-diffusivity scalar transport using a WENO scheme and dual meshing. J. Comput. Phys. 240, 158-173.

Lamont, J. C. \& ScotT, D. S. 1970 An eddy cell model of mass transfer into surface of a turbulent liquid. AIChE J. 16 (4), 513-519.

LEDWELL, J. J. 1984 The variation of the gas transfer coefficient with molecular diffusity. In Gas transfer at water surfaces, pp. 293-302. Springer.

Lewis, W. K. \& Whitman, W.G. 1924 Principles of gas absorption. Ind. Eng. Chem. 16.

Liu, X., Osher, S. \& Chan, T. 1994 Weighted essentially non-oscillatory schemes. J. Comp. Physics 115 (1), 200-212.

Magnaudet, J. 2003 High-Reynolds-number turbulence in a shear-free boundary layer: revisiting the Hunt-Graham theory. J. Fluid Mech. 484, 167196.

Magnaudet, J. \& Calmet, I. 2006 Turbulent mass transfer through a flat shear-free surface. J. Fluid Mech. 553, 155-185.

McCorquodale, M. W. \& Munro, R. J. 2017 Experimental study of oscillating-grid turbulence interacting with a solid boundary. J. Fluid Mech. 813, 768-798.

McKennA, S. P. \& McGillis, W.R. 2004 The role of free-surface turbulence and surfactants in air-water gas transfer. Int. J. Heat Mass Tran. 47 (3), 539-553.

NAGAOSA, R. 1999 Direct numerical simulation of vortex structures and turbulent scalar transfer across a free surface in a fully developed turbulence. Phys. Fluids 11 (6), 1581-1595.

NAgaosa, R. \& HANDlER, R.A. 2003 Statistical analysis of coherent vortices near a free surface in a fully developed turbulence. Phys. Fluids 15 (2), 375-394.

PAN, Y. \& BANERJEe, S. 1995 A numerical study of free-surface turbulence in channel flow. Phys. Fluids 7 (7), 1649-1664.

Perot, B. \& Moin, P. 1995 Shear-free turbulent boundary layers. Part 1. Physical insights into near-wall turbulence. J. Fluid Mech. 295, 199-227.

RodI, W. 2017 Turbulence modeling and simulation in hydraulics: a historical review. J. Hydraul. Eng. 143 (5), 03117001. 
Schwertfirm, F. \& MAnhart, M. 2007 DNS of passive scalar transport in turbulent channel flow at high Schmidt numbers. Int. J. Heat Fluid Flow 28 (6), $1204-1214$.

Shen, L., Yue, D.K.P. \& Triantafyllou, G.S. 2004 Effect of surfactants on free-surface turbulent flows. J. Fluid Mech. 506, 79-115.

Theofanous, T. G. 1984 Conceptual models of gas exchange. In Gas Transfer at Water Surfaces (ed. W. Brutsaert \& G.H. Jirka), pp. 271-281. Dordrecht: Springer Netherlands.

Theofanous, T. G., Houze, R. N. \& Brumfield, L. K. 1976 Turbulent mass transfer at free, gas liquid interfaces with applications to open channel, bubble and jet flows. Int. J. Heat Mass Tran. 19 (6).

Turney, D. E. \& BanerJee, S. 2013 Air-water gas transfer and near-surface motions. J. Fluid Mech. 733, 588-624.

VAriano, E.A. \& Cowen, E. A. 2013 Turbulent transport of a high-Schmidt-number scalar near an airwater interface. J. Fluid Mech. 731, 259-287.

Walker, D. T., Leighton, R. I. \& Garza-Rios, L. O. 1996 Shear-free turbulence near a flat free surface. J. Fluid Mech. 320, 19-51.

Wissink, J.G. 2004 On unconditional conservation of kinetic energy by finite-difference discretisations of the linear and non-linear convection equation. Comput. Fluids 33, 315-343.

Wissink, JG, Herlina, H, Akar, Y \& Uhlmann, M 2017 Effect of surface contamination on interfacial mass transfer rate. J. Fluid Mech. 830, 5-34.

Wissink, J. G. \& Herlina, H. 2016 Direct numerical simulation of gas transfer across the air-water interface driven by buoyant convection. J. Fluid Mech. 787, 508-540.

YANG, D. \& Shen, L. 2017 Direct numerical simulation of scalar transport in turbulent flows over progressive surface waves. J. Fluid Mech. 819, 58-103. 